\title{
No art without propaganda: la constitución de Nykino contra la institución documental*
}

NO ART WITHOUT PROPAGANDA: NYKINO'S CONSTITUTION AGAINST THE DOCUMENTARY INSTITUTION

NO ART WITHOUT PROPAGANDA: A CONSTITUIÇÃO DE NYKINO CONTRA A INSTITUIÇÃO DE DOCUMENTÁRIO

\section{Ana Rodríguez Granell**}

Cuadernos de Música, Artes Visuales y Artes Escénicas

/ Volumen 12 - Número 1 / Enero - Junio de 2017

/ ISSN 1794-6670/ Bogotá, D.C., Colombia / pp. 47-61

Fecha de recepción: 18 de mayo de 2016

Fecha de aceptación: 12 de agosto de 2016

Disponible en línea: 10 de marzo de 2017

doi:10.11144/Javeriana.mavae12-1.nawp

* Artículo de investigación. Estudios de Artes y Humanidades de la Universitat Oberta de Catalunya (Barcelona).

** Doctora en Historia del Arte por la Universidad de Barcelona. Profesora de los Estudios de Artes y Humanidades de la Universitat Oberta de Catalunya (Barcelona). 


\section{Resumen}

Este artículo analiza, a través de fuentes históricas y obras de historia cultural, la emergencia de colectivos de cine y documental militante durante el periodo del New Deal estadounidense teniendo en cuenta las tensiones entre estos y las políticas documentalistas institucionales 0 las directrices realistas de la década de 1930. Se plantea así que, ante la eclosión de nuevas tecnologías audiovisuales y la organización de redes de cooperación al margen de los estudios cinematográficos, algunas posiciones críticas no solo retan ciertas políticas estéticas hegemónicas, sino que también establecen nuevos diálogos con narrativas instituidas a la vez que retan los modelos de organización del trabajo de la industria cultural. Para ello, se tendrá en cuenta el caso de Nykino, como plataforma escindida que reformulará los preceptos del dogmatismo antivanguardista del Communist Party of the United States of America o la pretendida objetividad del documentalismo institucional que llevó a cabo una crítica del trabajo en los espacios de la militancia. De este modo, intentaremos insertar estas prácticas en el marco del modernismo estadounidense.

Palabras claves: militancia; trabajo creativo; realismo; documentalismo; New Deal; modernismo.

\section{Abstract}

This article analyzes through historical sources and works of cultural history, the emergence of collective film and militant documentary during the period of the New Deal period taking into account the tensions between them and institutional documentary policies or realistic guidelines of the thirties. It will explore how, the emergence of new audiovisual technologies, and the organization of networks of cooperation outside the studios, makes possible to understand critical positions that not only challenge certain aesthetic policies but also models of work organization. This will take into account the case of Nykino as split platform reformulating the precepts of antiavant-garde dogmatism of Communist Party of the United States of America or the alleged objectivity of the documentarism, conducting a critique of the creative work in the areas of militancy. Thus, we try to insert these practices within the framework of American modernism.

Keywords: militancy; creative work; realism; documentarism; New Deal; modernism.

\section{Resumo}

Este artigo analisa por meio de fontes históricas e obras de história cultural, o surgimento de filme coletivo e documental militante durante o periodo do New Deal, tendo em conta as tensões entre estas políticas e documentário institucional ou diretrizes realistas dos anos trinta. Ele irá explorar como, dada a emergência de novas tecnologias audiovisuais, ea organização de redes de cooperação fora dos estúdios, torna-se possível entender as posições críticas que não só desafiam certas políticas estéticas, mas também modelos de organização do trabalho. Isso levará em conta o caso de Nykino como plataforma de divisão reformular os preceitos do dogmatismo anti-avant-garde de Communist Party of the United States of America ou a suposta objetividade do documentário, a realização de uma crítica do trabalho nas áreas de militância. Assim, tentamos inserir estas práticas no âmbito do modernismo estadounidense.

Palavras chave: militância; o trabalho criativo; realismo; documentarismo; New Deal; o modernismo. 


\section{INTRODUCCIÓN}

Si entendemos el documental político como una respuesta crítica a los relatos de los medios de comunicación, podemos comprender el concepto de crítica como una práctica que atañe no solo al discurso contrahegemónico mantenido en los films, sino también como una reacción ante toda una serie de elementos que atraviesan la propia institución cinematográfica, como puedan ser la industria cinematográfica y sus correspondientes hegemonías productivas y estéticas o la propia institución documental y las políticas del realismo. Es decir, si consideramos el documental como expresión política, propongo explorar a qué dinámicas está respondiendo esta más allá del relato y cómo cuestiones como la propia tradición estética, las tensiones ante unas políticas culturales instituidas, la inmanencia histórica del debate cultural ante la idea de un arte político o la agenda política de determinado contexto social y geopolítico configuran las condiciones de emergencia y las tensiones que establecerán ciertas prácticas documentales de la militancia. De este modo, considerando la relación entre el documental y lo político como un dispositivo complejo, daremos cuenta de otra serie de coordenadas, por ejemplo las reflexiones en torno al trabajo, la resistencia a ciertas estéticas institucionalizadas o a determinados modelos productivos, que acaban por constituir una praxis crítica en el ámbito del cine militante.

Esta investigación analizará la configuración del colectivo Nykino y uno de sus films manifiesto Pie in the Sky (Steiner y Hurwitz 1935) y lo presentará como caso de estudio en los procesos de constitución de las estéticas políticas de la década de 1930 como respuesta a un marco sociocultural determinado y entendiéndolas como una práctica cultural situada. De este modo, se atenderá al marco y a la agenda de la política institucional en el periodo del New Deal (1933-1938), a la propia tradición cultural y a la emergencia del discurso modernista "factualista", así como a las propuestas de la institución documental, desde el documental gubernamental del New Deal hasta el noticiero obrerista del Communist Party of the United States of America. Así veremos cómo todos estos planos circunscriben la postura crítica y diferencial de un colectivo como Nykino y su capacidad de agencia ante ciertas dinámicas documentalistas e industriales. Cronológica y conceptualmente, nos ubicaremos en territorios poco explorados, como los momentos intersticiales en los que se fraguaron diversos debates en torno al documental político en los Estados Unidos y que en 1937 darían lugar al proyecto Frontier Films y su estética dialéctica (Alexander, 1975), con las conocidas cintas involucradas en el Frente Cultural o en las luchas por los derechos y las libertades civiles, como Native Land (Ralph Steiner, Willard van Dyke, Paul Strand, Leo Hurwitz, 1937-1942). 


\section{DOCUMENTALISMO INSTITUCIONAL: LA PROPAGANDA CONTRA LA PROPAGANDA DURANTE EL NEW DEAL (1933-1938)}

El gran movimiento documental de la década de 1930 estadounidense se puede comprender a partir de la expansión del discurso realista. Nociones como factual o documentary se extienden por el campo de las artes visuales, el teatro, la danza o la fotografía. Un especial interés por lo no ficcional al que se suman revistas como Life o Fortune con reportajes de Walker Evans y James Agee (Corbus 1999) o dando lugar a la crudeza del hard-boiled, toman gran empuje las formas literarias del testimonio y la autobiografía o el periodismo radical de John L. Spivak, mientras acontecía la representación de nuevos sujetos políticos en el nuevo realismo literario de este periodo (Sttot 1986; Rabinowitz 1991, 1994; McCann 2000; Szalay 2000). Incluso las ciencias sociales se vieron impulsadas por el ímpetu factualista, con el intento de articular la base de una democracia moderna donde todo ciudadano, como un ser racional, tenía igual acceso a los hechos — the facts - y podía actuar en consecuencia, inteligentemente, en la criba de los asuntos tanto públicos como privados.

Ello respondía a varios fenómenos que eclipsan el debate cultural y político a partir de la Gran Depresión y que en contexto global dialoga con la vuelta al racionalismo tras la primera posguerra, con la Nueva Objetividad en la República de Weimar, por ejemplo. Por un lado, a finales de la década de 1920, en los Estados Unidos se hizo necesario y urgente generar un aparato informativo frente a las carencias de una esfera pública que estaba dando la espalda a los problemas sociales emergidos con la crisis. El desarrollo tecnológico cristalizado en el acceso a aparatos fotográficos y de filmación supuso una apertura de esa esfera pública. Por el otro lado, las instancias gubernamentales sostenían una comunicación política nutrida de prácticas documentales basadas en un mito verista-humanista. De este modo, la definición de lo documental, lejos de esclarecerse o sostener algo cercano a la neutralidad, se tornaría un receptáculo de múltiples discursos atravesados ideológicamente.

Engarzado en estas disputas, el debate en torno al realismo como herramienta moderna de gobernabilidad y cohesión social —en parte imitando el proyecto documental griersoniano— nos sitúa ante la preocupación del Gobierno de Franklin D. Roosevelt respecto de la función social de los medios de masas. El mismo programa político del New Deal se presentó como un moderno programa tecnocrático - aunque innovador en su vertiente filosocialista - y despojado de ideología ante la amenaza del bolchevismo y contra la efusiva estetización política de los fascismos europeos. Así, los nuevos formatos editoriales y fotográficos afines al proyecto político se presentaban bajo el titular de verdadera naturaleza de los hechos o documentos humanos (Sttot 1986; Böger 2001; Rabinowitz 1994).

Ante la crisis de la democracia liberal y ante el racionalismo instrumental de la predecesora legislación de Herbert Hoover (1920-1933), la comunicación política de Roosevelt se articulará respecto de afectividad y humanismo (Sttot 1986, 11). Con vistas a sortear la ofensiva conservadora anticomunista, el documentalismo afectivo que apelaba al American People se teñía de una paradójica idea de una propaganda contra la propaganda. Así aparecieron, por ejemplo, los sondeos America Speaks, las metodologías del American Institute of Public Opinion o el Institute of Propaganda Analysis de Clyde Miller y la sociología crítica (Sproule 1997, 131). En ese marco de ingeniería social, se insertarán programas de políticas públicas, como la Work Progress Administration y la Farm Security Administration, que financiarían parte de los proyectos del documentalismo estadounidense o estudios sobre cultura popular, como los Middletown Studies de Robert Wilson Lynd. 
Contando con artistas y sociólogos, el fomento de esa expresión documental pretendía conectar con la idiosincrasia demócrata local gracias al sometimiento controlado de los implicados para evitar que, como comentara Roosevelt, ningún entusiasta pintara el busto de Lenin en el Palacio de Justicia (Denning 1996, 45). De este modo, por primera vez, se genera una política cultural que dará lugar a cierto cine documental institucional, en el que cabría incluir cintas como The Plow That Broke the Plains (1936) o The River (1937), de Pare Lorentz, producidas por la Resettlement Administration y la Farm Security Administration, respectivamente. La Resettlement Administration de Roosevelt y la pléyade de intelectuales del servicio cultural del New Deal pusieron en manos de Pare Lorentz la realización de The Plow, a fin de informar sobre las medidas del Gobierno para realojar a los granjeros afectados por la gran nube de polvo. La mirada crítica que se le suponía a la nueva expresión documental no era demasiado rigurosa si se trataba de promocionar la política de las tres R: relief, recovery y reform. Desde el punto de vista del contenido, el film de Lorentz no generaba ningún conflicto sobre el asunto de las grandes llanuras ni ponía en duda el discurso oficial y neutralizado, sino que generaba una mera descripción del desastre natural. Desde el punto de vista formal, la factura del documental es muy similar a las propuestas del entorno griersoniano: grandes planos sobre las llanuras y una voz en off de tono épico que describía las dificultades a las que se enfrentan sus habitantes, su éxodo, la subida de precio del trigo a causa de la guerra. Una música melancólica acompaña todo el pasaje y descuida ofrecer datos o información sobre las medidas del Gobierno. Tampoco aparecen referencias a las causas originarias del desastre ecológico por sobreexplotación y aparecen como únicos responsables los ciclos climáticos de sequías (Ellis y MacLane 2005, 181).

Del mismo modo actuaba el segundo documental The River. Financiado por la Farm Security Administration generaba conciencia sobre la preservación de los recursos naturales y la construcción de presas en el Mississippi en un tono afirmativo (tarea llevada a cabo por Tennessee Valley Authority). Junto con el propósito de informar a la población sobre los planes de recuperación, también existía la necesidad de contrarrestar las críticas del núcleo conservador del Congreso frente al supuesto "socialismo" que se desprendía de las medidas "prosoviéticas", como la implantación de cooperativas agrarias para el campesinado. De nuevo, en un tono celebratorio y épico sobre las gentes y los recursos naturales de los Estados Unidos, las tomas se superponen a la voz sobreactuada del tipo The March of Time y una sinfonía grandilocuente (Virgil Thompson), aunque esta vez sí se apuntan los logros conseguidos a través de las medidas del New Deal (nuevas plantaciones, mejora en la maquinaria agrícola, implantación de granjas modernas...), a la vez que se contrarrestan con los desastres naturales y desbordamientos del río. The River será premiado en el Festival de Cine de Venecia de 1938, nominado al Premio Pulitzer por la narrativa poética y exhibida en más de cinco mil salas, debido a que fue uno de los pocos documentales que tuvo la suerte de que una major, la Paramount en este caso, se ocupara de la distribución (Ellis y MacLane 2005, 84). Tal éxito le valdría a Lorentz la fundación de la United States Film Service y la producción del docudrama promocional para la Public Health Service The Fight for Life (1940). 


\section{FUERA Y CONTRA LA INSTITUCIÓN: EL DOCUMENTAL PROLETARIO DE WORKERS FILM AND PHOTO LEAGUE}

Paralelamente, tal y como hemos comentado, el auge del marxismo y los ecos del comunismo en un país donde las luchas sindicales tuvieron un alcance mayor que en ningún otro lugar, derivó en la diseminación de plataformas de comunicación alternativa, como las ligas de fotografía y de cine obrero, en consonancia con otros núcleos europeos y soviéticos (Ribalta 2011). Ya desde los inicios del siglo XX, el uso contestatario del cine marcó dos periodos previos de documentalismo proletario y de ficción, que abarcan desde 1907 a 1920 (Ross 1999; James 2005). Sin embargo, tras el auge del conservadurismo, la oleada internacionalista abrirá un tercer periodo de cine hecho desde y para la clase obrera basado en las redes de cooperación y asistencia logística, como federaciones y asociaciones. De este modo, los comunistas estadounidenses, en alianza con la Internationale Arbeiter-Hilfe germano-soviética, crean su propia sede de la International Workers' Aid en la década de 1920 en Chicago (Sweet, Rosow, Francovich y Brandon, 1973). En 1930, tras la experiencia de la Workers' Camera League, ante la situación de hegemonía del monopolio cinematográfico de los estudios hollywoodienses y como acción frente a los mecanismos de propaganda de la década de 1920 y a la ausencia absoluta de noticias acerca de los conflictos sociales en los medios de comunicación, se crea la Workers' Film and Photo League (WF\&PL) fundada por Robert del Duca, Lester Balog, Sam Brody y el crítico Harry Alan Potamkin, formada con obreros operarios y white collars (Nichols 1972, 108-115).

No exentos de represión policial a causa de la exhibición de películas prohibidas, la WF\&PL funcionaba gracias a una base logística para la filmación de noticieros obreros centrados principalmente en las luchas de clase (huelgas, marchas, sindicalismo, etc.). Con la aparición de la cámara Eyemo portátil, se hacía más fácil este tipo de prácticas documentales, gracias a la asistencia técnica y material de los asociados para la filmación de movilizaciones o para pases de películas en circuitos no comerciales, como sindicatos, centros educativos, asociaciones, parroquias o zonas rurales. La Liga estaba apoyada, además, por estructuras especializadas, como la distribuidora de films soviéticos Garrison Films y el cineclub Film Forum, donde el cineasta y teórico Jay Leyda programaba sesiones dedicadas al cine experimental y obrerista. La principal sede en número de asociados era la de Nueva York, seguida de la de Los Ángeles, junto con otros núcleos activos que se ubicaban en Detroit, Hollywood, Chicago, Boston y San Francisco.

La existencia de la WF\&PL, que perdurará hasta 1935, cobró mayor sentido cuando la legislación Hoover aún no había reconocido públicamente el calibre del desastre de 1929 y en un momento en el que la esfera pública no respondía ante la estrepitosa situación de crisis; no será hasta 1933 que el Gobierno de Roosevelt haga públicas las primeras estadísticas de desempleo (Sweet, Rosow, Francovich y Brandon 1973, 13). Según comentó posteriormente Hurwitz $(1975,13)$, mientras las calles se veían invadidas de gente sin hogar y sin trabajo, los cines seguían pasando las mismas películas del tipo chico-conoce-chica mientras los medios de comunicación daban la espalda a la situación real que se vivía en el país.

Casi la totalidad de films de la Liga se realizaron por asociados en el ámbito del newsreel (Alexander y Campbell 1977), algunas de ellas: Detroit Ford Massacre, Hunger March (19311932), Hunger (1931), Bonus March, May Day (1934). 
Hemos de entender la WF\&PL como un caldo de cultivo donde se empieza a gestar una comunidad política de izquierdas al margen de los estudios, que tuvo posibilidad de formarse autodidácticamente leyendo la revista británica Close Up. La estructura de la Liga se sostenía a base de voluntariados que ofrecían clases de cine a los miembros, con las aportaciones monetarias o películas vendidas a bajo coste por gente próxima al grupo. Por su parte, lo que diferenciaba a la WF\&PL de otros grupos similares en contextos como el británico fue justamente la radicalización militante. A diferencia de la independencia poco definida de la tendencia vanguardista del Art Cinema, o de movimientos como el documentalismo de John Grierson (Aitken 1992; Sexton 2008), quien participó en la fundación de las Workers' Films Societies británica, la Liga estadounidense optó desde el principio por "la dura vía de la independencia", tanto en subsidios o encargos estatales como en relaciones comerciales con la industria (Sweet, Rosow, Francovich y Brandon 1973, 16), negando el trabajo en la industria cinematográfica como vía de sustento subsidiario al trabajo militante (Alexander 1981, 45). Una posición política que se mantendrá férrea en la escisión de Nykino.

Por otro lado, la apuesta estética de la Liga se planteó en torno a dos cuestiones: bien registrar las luchas diarias, bien salvar las dificultades productivas para estos grupos, desarrollar y explorar nuevas narrativas de ficción que podían implicar el trabajo con actores o medios más costosos, iniciativa esta que retrasaría y limitaría la asistencia al acontecer diario del movimiento obrero (Sweet, Rosow, Francovich y Brandon 1973, 15). Finalmente, dada la intensidad del momento, el estandarte de la Liga se centró en el cine directo proletario y la realización de noticieros ${ }^{1}$. Pero esta postura no solo fue escogida por motivos de precariedad, sino que la política del grupo se veía afectada también por los dictados provenientes del aparato comunista. Dadas sus vinculaciones con la Internacional Comunista, la filmación de noticieros se convertirá en una forma segura de evitar cualquier debate en torno a desviaciones vanguardistas. Recordemos que, tras el ascenso de Stalin en 1929, el Partido fue imponiendo la hegemonía de la Asociación Rusa de Escritores Proletarios que marginó cualquier atisbo de experimentación formalista y se agudizó con la llegada de Andrey Aleksandrovich Zhdanov a la Unión de Escritores Soviéticos en 1932 (Clarck 2000).

Ya desde las filas de la WF\&PL existían ciertos resquemores entre aquellos partidarios del modo artístico: Leo Hurwitz, Ralph Steiner (que había realizado algún film experimental como H2O en 1929) e Irving Lerner; y los más alejados de este, Sam Brody, Leo Seltzer, Kern, Del Duca, Kandell, Roffman, Tom Brandon y David Platt. Pero también otros conflictos de tipo político atravesaban la League. Si bien el marxismo de sus componentes era algo en formación y muchos no estaban adscritos a la idea de una política revolucionaria, hacia 1931 se había puesto en juego la creación de un frente cultural más fuerte liderado por la línea dura del Communist Party of the United States of America. De este modo, se comenzaron a generar procesos de alineación al partido por parte de miembros de la Liga a través de la creación del Workers Cultural Federation y siguiendo el dirigismo político de la Unión Internacional de Escritores y Artistas Revolucionarios del Komintern.

No obstante, emergerán multitud de voces críticas ante la situación soviética. El historiador William Alexander $(1977,46-47)$ cita el caso del miembro de la Liga, Isador Lerner, que, tras un viaje a Ucrania y haber experimentado las carestías sociales de la región, expresó sus desavenencias con un sistema que parecía no funcionar o que, al menos, no concordaba con las noticias recibidas desde el otro lado del Atlántico. Inmediatamente, Lerner fue expulsado por reaccionario. Las tendencias puristas de la Liga persistirán hasta el Frente Cultural de 
1935 con posturas antiindividualistas que impedían cualquier atisbo de autoría por parte de los realizadores. Cineastas como Leo Seltzer o Lewis Jacobs -y si hubiera vivido, seguramente también el teórico Allan Potamkin - serían algunos de los afectados por esta línea política. Por su parte, Potamkin ya había anunciado en uno de sus manifiestos, Workers Films (1930), que el documental era solo el primer paso para lo que debería ser, en última instancia, el "film dramático de contenido revolucionario" (citado por Campbell 1978, 23-28).

Así, frente a la postura de Potamkin, estaba la de Sam Brody quien defendía la idea de un cine proletario solo deseable a través del documento directo $y$, obviamente, contra cualquier colaboración con las instituciones estatales. El film como documento verídico solo es posible mediante tres formas, según Brody: 1. factual film, 2. newsreely 3. film sintético; el lema era sin actores, sin artificio, sin ilusionismo ${ }^{2}$.

Como veremos, la fracción de algunos cineastas pretendieron reafirmar el diálogo entre el arte y lo político, y a la contra, tanto del pretendido objetivismo institucional como de las sobrias políticas de la WF\&PL, recuperar ciertas fórmulas narrativas para la construcción de sus discursos críticos.

\section{LA ESCISIÓN NYKINO (1934-1937): ARTE Y PROPAGANDA PARA EL DOCUMENTAL POLÍTICO}

Nykino fue fundada en 1934 por los miembros de la Liga: Leo Hurwitz, Ralph Steiner, Lionel Berman, Sidney Meyers e Irving Lerner. Algunos visionados de películas extranjeras cercanas a la vanguardia animaron al grupo a generar este nuevo movimiento de oposición: Pomme de Terre o Prix et Profits (Yves Allégret y los hermanos Prévert, 1932) y Tres cantos a Lenin (Tri pesni o Lenine, Dziga Vertov, 1934) fueron los casos más citados; mientras que desde 1933 ya habían puesto en práctica sus ideas a través de un pequeño grupo de cine experimental con la Workers Laboratory Theatre y el Group Theatre de Nueva York. La idea de fundar un movimiento cinematográfico cobró más urgencia tras la firme posición de la Liga en la League's National Film Conference de no permitir ninguna variación más allá de la producción de newsreels (Hurwitz 1975, 10).

La proclama más conocida de los disidentes sería No hay arte sin propaganda y No hay propaganda efectiva sin arte (Hurwitz 1934, 14-15). Podemos considerar que estos reclamos contienen una doble posición política. Por un lado, Nykino se situaba contra el dogmatismo newsreel de la League contra cualquier atisbo artístico, mientras que, por otro, arremetía contra la pretendida neutralidad del documentalismo institucional y abogaba por la necesidad de un cine de agitación y propaganda. Nykino siguió produciendo noticieros, pero ahora su postura política residía en reconocer la capacidad de agencia de las formas hegemónicas de comunicación. Es decir, las formas estéticas promovidas por el grupo, lejos de postularse antagónicas al vilipendiado The March of Time, abogaban por el mismo uso de sus fórmulas dramáticas. Mientras el noticiero de Time Inc. utilizaba una propaganda reaccionaria, el noticiario de Nykino, The World Today, lo hacía en sus mismas formas, pero con un contenido progresista: "Así, la película iba a tratar de acercarse a sus temas, a su gente, y teatralizar los acontecimientos a través de la invención de circunstancias y actividades" (Alexander 1977, 190).

Otras fuentes de inspiración para su programa estético serían el film New Earth (Nieuwe Gronden, Joris Ivens, 1933) y la película de ficción y propaganda Frontier (Aerograd, Alexandr Dovzhenko, 1935), que daría nombre a su ulterior formación frentepopulista Frontier Films en 
1937 (García López 2013, 56). De hecho, el mismo Joris Ivens y reconocidos cineastas de la vanguardia como Paul Strand se afiliarán al grupo de Nykino a partir de 1935. Las afinidades entre Ivens y Nykino en torno a la naturaleza propagandística del documental iban en la misma dirección en cuanto, comentaba Ivens:

Por supuesto, que nos marcaron inmediatamente como propagandistas, y de tal manera, que aceptamos esa etiqueta. En cierto modo, es cierto... Creo que acerca de estos grandes problemas como la vida y la muerte y la democracia y el fascismo, no hay objetividad posible para un artista [...] Su trabajo tiene que ser muy emocional. Creo que se podría decir que el cine documental es una presentación emocional de los hechos. El público puede tratar de ser objetivo, pero no el director de cine documental ${ }^{3}$.

Por su parte, Paul Strand, tras sus éxitos a través de films de vanguardia como la sinfonía urbana de Manhatta (1920), había conseguido su prestigio gracias al pseudodocumental rodado en México Redes (The Wave, Fred Zinnemann, Emilio Gómez Muriel, 1937), cuyo proyecto rehusó tanto los métodos del film institucional instructivo como las formas excesivamente experimentales y buscaría, a través de las propuestas de Robert Flaherty, un tipo de lenguaje documental apropiado para el gran grueso de la población mexicana (Alexander 1975, 18).

Al margen de consideraciones estéticas, otro de los objetivos marcados por la escisión de la Liga fue el de superar aquella precariedad, formal y profesional que caracterizaba la improvisada esfera pública obrera, y la falta de rigor en la producción independiente de films de ficción tan criticada por Hurwitz, Lerner y Steiner; estas películas habían sido realizadas por algunos miembros de la League apartados de Brody: Sheriffed, de Nancy Naumburg y James Guy (1934); The New Legion, de Irving Browning y Manon Miller (1934); y On the Waterfront, de Ed Kern y Leo Seltzer (1934). Por su parte, la Harry Alan Potamkin Film School sería un punto logístico de apoyo más.

Las películas de Nykino funcionaban, pues, como manifiesto crítico a las limitaciones del cine proletario anterior. Entre ellas podemos señalar la inacabada y antibélica Café Universal (Ralph Steiner, 1934) donde actuó Elia Kazan y Art Smith; Harbor Scenes, G v ranite o Quarry (Ralph Steiner, 1936); y, sobre todo, la película completamente alejada ya del noticiero proletario Pie in the Sky (Steiner, 1935). Todas ellas, como también la formación del grupo, seguían manteniendo la completa independencia respecto de Hollywood y de las instituciones estatales. Al respecto, Elia Kazan, miembro de Group Theater, señalaba: “Teníamos la determinación de cambiar América y convertirla en socialista. Mientras tanto, ¡Roosevelt —nuestro mejor amigo- destruía nuestro programa poniendo en marcha el capitalismo" (Kazan y Ciment 1987, 28).

Como veremos a continuación, lo interesante de Nykino va a ser ese doble proceso de reflexión crítica: uno basado en la conciencia del lenguaje cinematográfico y la efectividad de una tradición estética popular, tal y como sucede en el inusual film de género Pie in the Sky; y otro, el de llevar el trabajo voluntario de la militancia al terreno de la creación artística. 


\section{PIE IN THE SKY (STEINER, 1935)}

Pie in the Sky, realizada en colaboración con el Group Theater, se pensó como una sátira contra el dogmatismo religioso. Este film, a nuestro modo de ver, representa uno de los experimentos más innovadores de la filmografía de izquierdas en el periodo de entreguerras y realizada al margen de las instituciones. A través de un corto cómico, género inusual en este tipo de realizaciones, este film de ficción todavía mudo presenta bajo una estructura narrativa y un uso rítmico del montaje la recreación ilusoria de los dos mendigos que deambulan en un vertedero tras haberse quedado sin su trozo de tarta en el centro de beneficencia. Los dos personajes inventan una Mae West con un viejo maniquí, un viaje en coche en un chasís desgajado... hasta que la trama imaginada se convierte en la trama del propio film que diluye la frontera entre fantasía y realidad. Los hambrientos personajes acaban en un imaginado departamento de asistencia social donde, muertos de hambre y a través de un sacerdote, intentan invocar al Señor para hacerse con un trozo de tarta; secuencia amenizada que mezcla planos fijos y recrea la canción popular que da nombre a la película: "You will eat, by and bay, in the glorious land above the sky. Work and pray, live on hay, you will get pie in the sky when you die". La película suponía un desafío no solo a las formas usuales de la propaganda de izquierdas, sino que presentaba una crítica social a través de elementos metanarrativos.

A pesar de una frescura que acercaría la película a las realizaciones populares, esta fue duramente criticada y marginada por los mismos núcleos del cine proletario. Por ejemplo, Ray Ludlow en New Theater criticaría que tal sátira sobre la Iglesia no podía más que tirar piedras contra la audiencia potencial de Nykino (Alexander 1981, 63). En cualquier caso, con el paso del tiempo, se señalaría este film como un producto adelantado a su época. Según el militante Tom Brandon: "Pie in the Sky apareció cerca de 30 años antes de las interesantes películas de improvisación de John Cassavetes" (Sweet, Rosow, Francovich y Brandon 1973, 20).

\section{CRÍTICA A LOS MODELOS DEL TRABAJO CREATIVO}

Tal y como se ha señalado, la militancia de Nykino intentaría ir más allá de la elaboración de una estética política y propone una reflexión sobre las condiciones laborales del trabajo creativo tanto en entornos de militancia como rechazando las formas de organización fordista de la industria cultural. Tal y como funcionaba el grupo de agitprop Shock Troupe desde el Workers Laboratory Theatre, la idea era generar un grupo cooperativo totalmente cohesionado y dedicado plenamente a la producción cinematográfica de agitación que trabajaba de diez a doce horas diarias (Hurwitz 1934, 23).

El acercamiento al Group Theater de Nueva York liderado por Lee Strasberg, Harold Culman y Cheryl Crawford ${ }^{4}$ y germen del Actors Studio (en 1941), aparte de introducir el método Stanislavsky basado en la recuperación de la memoria afectiva del actor para reproducir un mayor verismo en las representaciones, destacó por insertar un nuevo sistema de organización en la compañía: los sueldos se uniformaban y no se pagaba a los actores en función de su más o menos protagonismo, sino por su capacidad de involucrarse en el grupo y sus necesidades familiares. Esta, entre otras reformas, pretendía alcanzar una expresión común, animando y respetando todas las aportaciones individuales de modo que se generase una compañía fruto del trabajo colaborativo, pero, y esto era relevante, sin abatir la vocación y creatividad individuales. 
La reflexividad sobre cuestiones organizativas, laborales, afectivas, y la situación del trabajo creativo dentro de estas, respondía críticamente a un modelo productivo determinado representado por Broadway o Hollywood. En líneas generales, este modelo hegemónico limitaba las capacidades creativas de los agentes implicados en la producción del film que era sometida a una especialización por áreas que jerarquizaba y instituía las fases de realización de acuerdo con las directrices definidas por una línea de producción, presupuestos, distribución y exhibición, diseñada por la cúpula directiva instalada en Nueva York. Del mismo modo que en la WF\&PL, la Nykino mantendría su militancia en la negativa a trabajar para la industria cinematográfica y retaba la común subordinación estructural del artista. De hecho, esta autoconciencia del trabajador cultural ante la organización fordista del trabajo no será una posición exclusiva de las organizaciones de izquierdas y, desde el seno de la industria en 1933, se funda el Screen Writers Guild. Aunque ya había habido tentativas previas en 1914, estas habían fracasado dado que la Academy of Motion Pictures Arts and Sciences había dominado las relaciones laborales de guionistas y productores entre 1927 y 1933. En realidad, la emergencia de organismos sindicales informales en Hollywood antecede a la oleada crítica de la década de 1930: en 1919, tuvo lugar la primera huelga de trabajadores de Hollywood y multitud de asociaciones habían emergido frente a las abusivos contratos con las productoras, desprotegidos de leyes federales que no se aplicaban a la industria cultural y con pocas posibilidades de enfrentarse a una organización vertical de los estudios. De este modo, en 1933, y frente a los recortes de $50 \%$ del salario a los trabajadores de la industria, se lleva a cabo la formación del sindicato independiente Screen Writers Guild con John Howard Lawson a la cabeza (Staiger 1979; Schwartz 1982).

Por su parte, los integrantes de Nykino sostenían la radicalidad de la época de la Lega, tenían como forma de sustento oficios externos al cinematográfico o estaban en talleres ocupacionales, mientras que los más afortunados eran fotógrafos en revistas como Harper's Bazaar. El grupo se sentía cohesionado tanto por las condiciones sociales como por la ideología compartida. Ambos factores se retroalimentaban mutuamente: se trataba de una militancia costosamente radicalizada, puesto que afectaba directamente a sus circunstancias vitales. Una situación difícil la de los componentes de Nykino, si se sumaba el carácter de rojos, emigrantes, judíos, parados y artistas. Y esto último era algo de lo que se debía prescindir si se pretendía acceder y permanecer en un marco comercial. Podríamos decir al respecto que se trataba de una conciencia de institución antiinstitucional, tal y como Pierre Bourdieu analizaba en el caso del campo artístico decimonónico (1995, 47 y 383). Incluso, en algunas cintas cinematográficas del periodo antifascista de Frontier Films (como Native Land), empiezan a cobrar fuerza ideas más allá de la condición de clase, como eran las cuestiones raciales y étnicas que suponían una reconfiguración de la agenda política desde los movimientos sociales.

En cuanto al modelo económico de Nykino, esta seguirá dependiendo de aportaciones individuales, colaboraría con organizaciones obreras y recaudaría fondos a través del alquiler de copias y exhibiciones en centros sociales, sindicatos, etc. Por tanto, con una clara voluntad de generar modelos alternativos de financiación al margen de la industria.

Nykino puede considerarse como un periodo de transición hacia la compañía Frontier Films (1937-1942) tras la incorporación de Paul Strand en 1935 y Joris Ivens en 1936 enmarcado en el auge frentepopulista del Frente Cultural (García López 2013). La evolución de la Nykino hacia la Frontier pasará tanto por cuestiones estéticas como organizativas. En el modelo productivo, se dará el salto al diálogo, no con Hollywood, pero sí con instituciones de nuevo mecenazgo, como la Whitney o la Robert Marshall Foundation (en un momento en el que Jay Leyda 
dirige el área cinematográfica del Museum of Modern Art [MoMA]). Igualmente, se suavizaron posturas antigubernamentales y se establecieron vínculos con la Administración Roosevelt a través de Pare Lorenz en el equipo de The Plow that Broke the Plain (1937) (anteriormente Steiner ya había colaborado en proyectos del New Deal con Hands [1934]).

En lo estético, el interés por la obra teórica de Vsevolod Illarionovich Pudovkin sobre técnica cinematográfica (1980 [1928]) y las formas narrativas del montaje, lejos del uso efectista, elaboraron un retorno a las formas clásicas. Es decir, siguiendo a Pudovkin, la idea era presentar al espectador una trama coherente y una narración clara que lo involucrara en los acontecimientos.

En definitiva, la crítica a Hollywood y sus modelos sigue presente; sin embargo, tal como Ivens publicó en uno de sus primeros artículos contra la industria cinematográfica en New Theater ${ }^{5}$, la posición política de la militancia pasaba por la necesaria organización de estudios independientes para tratar el cine con una nueva vitalidad. De esta forma, comenta (2000, 20-21), Ivens y el grupo de Nykino representaron un cambio de rumbo en la factura formal del documental hacia una estructura dialéctica que combina la dramatización y la imagen documental presentando los acontecimientos mediante la fórmula conflicto-resolución-mirada al futuro6 ${ }^{6}$

\section{CONCLUSIONES}

Tal y como apuntaron inicialmente Steiner y Hurwitz en "A New Approach to Film Making" (1935), el impacto y las formas experimentales de la vanguardia no tenían por qué ser rechazadas en una nueva estética documental que incorporase estructuras dramáticas convencionales. De la incorporación de postulados como la "Avant-garde documentary film" de Ivens y el trabajo en Nykino a partir de estructuras narrativas clásicas y un interés por los géneros populares se puede señalar que, en cierta forma, estas propuestas documentales, se pueden considerar como una apuesta moderna más en la superación de la dicotomía vanguardia/realismo al proponer una reconfiguración de los límites de la propia representación institucional.

No bastaba con filmar eventos revolucionarios, sino que la imagen debía explicar las fuerzas que los suscriben y, para ello, era necesario adoptar formas narrativas que permitieran una implicación con el relato. En este sentido, estos artistas no están tan lejos de las formas en cómo estaba operando Hollywood al instituir un modo de representación "naturalista" basado en el centramiento del espectador.

Tal y como suscribía Pie in the Sky, el juego con los géneros clásicos populares y la presencia de componentes cinematográficos metadiscursivos rechazaron la negación radical de la vanguardia para insertarse en una apertura del realismo hacia nuevas formas de lo decible y a través de la vía política. Considerándolo así, podríamos incluir estas prácticas documentales en el llamado modernismo estadounidense de la década de 1930, cuya especificidad cultural se basó en elaborar un movimiento vanguardista propio que superase el escollo de cierto elitismo europeo de la alta cultura hacia vías más populares y que fue impulsado, como comentábamos al inicio, por todas estas prácticas ligadas a las mejoras tecnológicas de grabación y reproducción audiovisual. Asimismo, podemos considerar tanto la praxis como las relaciones de los integrantes de Nykino con la teoría cinematográfica como un pleno posicionamiento moderno. La conciencia ante las propias condiciones sociales del artista o su capacidad reflexiva ante el lenguaje instituido del cine que incorporan en su propia praxis nos puede llevar

a plantear la historia del cine antes de la eclosión de prácticas radicales durante la década de 1970, como un periodo también contenedor de una agenda política propia y ajustada a sus 
propias condiciones históricas. Unas condiciones, por otro lado, que nos permiten analizar las reestructuraciones de los modelos y las políticas de producción cultural cuando se proponen estudios comparativos entre aquellos casos cinematográficos sometidos a la crisis mundial de la década de 1930 y, por ejemplo, movimientos sociales emergidos a causa de la crisis de 2008 y que han impulsado la práctica de cine y video militante con el apoyo de las herramientas digitales. Si bien las demandas de Nykino pasaban por entender el trabajo militante fuera de los márgenes de la industria fordista, hoy podemos estudiar prácticas audiovisuales en un marco nuevo donde el trabajo flexible y la autoprecarización ligada al trabajo creativo en la economía digital imponen nuevas metas políticas ligadas al paisaje del posfordismo. De modo que no solo las temáticas del discurso crítico audiovisual están sometidas a las urgencias del momento (reivindicación de derechos básicos como el acceso a la vivienda o los efectos de terciarización de las ciudades), sino que también las propias condiciones sociales de los creadores se incorporan en la praxis audiovisual a través del uso de licencias libres que hoy se van incorporando poco a poco en el sistema de difusión cinematográfica, como festivales legitimados o plataformas de video on demand que realizan films de forma cooperativa y autofinanciada y acceden tímidamente a los circuitos comerciales y proponen espacios de negociación y diálogo entre el activismo político y las estructuras clásicas de los medios de comunicación, como televisiones y distribuidoras cinematográficas.

\section{NOTAS}

1 Los medios de difusión que daban soporte a la Liga fueron las revistas especializadas New Theater editada por Herbert Klein —órgano también de la Worker's Theater o la Workers Dance League_y Experimental Cinema o Film Front que divulgaban a su vez el repertorio de cine soviético, así como otras publicaciones marxistas incluirían numerosos artículos al respecto, como New Masses o el Daily Worker, revista y diario oficiales del Communist Party of the United States of America.

2 Según el manifiesto publicado por Brody en New Theater de 1934 "The Revolutionary Film: Problem of Form", a lo que Hurwitz respondería con el texto "The Revolutionary Film-Next Step" (Campbell 1982, 115-117).

3 Palabras expuestas en la conferencia de Ivens "Documentary: Subjectivity and Montage" para el MOMA en 1939 (Bakker 1999, 250-260)

4 La escena teatral estadounidense se caracterizaba, ya desde el auge de los magnates teatrales de Broadway a principios de siglo $X X$, por toda una serie de iniciativas no profesionales que luchaban por ofrecer un repertorio propio, artístico y, a veces, politizado frente al teatro comercial. Esto marcó un nuevo periodo teatral en la década de la Primera Guerra Mundial, con el éxito comercial de compañías como el Theater Guild, aunque este acabaría siguiendo los pasos del teatro convencional de Broadway. La aparición del Group Theater marcó otro hito por la introducción del teatro moderno en los Estados Unidos a través de textos de Chéjov o Strindberg (Carpiolo 1968).

5 Se trata del texto "Notes on Hollywood" en New Theatre, $n^{\circ} 3$ de 1936 (citado por Schoots 2000, 113). Del contexto hollywoodiense Ivens admiraba, a pesar de los límites impuestos por la industria, a Capra, Mamoulian, Milestone y Vidor y películas como El delator (The Informer, J. Ford, 1935), Tiempos modernos (Modern Times, Ch. Chaplin, 1936), El secreto de vivir (Mr. Deeds Goes to Town, F. Capra, 1936), Furia (Fury, Fritz Lang, 1936) o La tragedia de Louis Pasteur (Pasteur, W. Dieterle, 1935).

6 Para Costa (1999), el film representa, por un lado, la superación de los métodos constructivistas de la vanguardia soviética; y por otro, la introducción de una nueva reflexión sobre la imagen documental donde el estilo personal se elabora a partir de una dialéctica frontal con los acontecimientos reales, tal y como supuso la filmación en el frente de la guerra civil española en The Spanish Earth (J. Ivens, 1937). Una revolución representacional que, para Costa, antecede a la siguiente transformación que tendrá lugar con el uso del sonido directo de las cámaras ligeras en la década de 1960 con Le 17ème Parallèl: La guerre du peuple (1968) del mismo Ivens. 


\section{REFERENCIAS}

Aitken, Ian. 1992. Film and Reform: John Grierson and the documentary Film Movement. Londres: Routledge.

Alexander, William. 1975. "Frontier Films, 1936-1941: The Aesthetics of Impact". Cinema Journal 1, n. ${ }^{\circ} 15: 16-28$.

- 1977. "The March of Time and the World Today". American Quarterly 2, n. ${ }^{\circ}$ 29:182-193.

- 1981. Film on the Left, American Documentary Film from 1931 to 1942. Princeton: Princeton University Press.

Alexander, William y Russell Campbell. 1977. "Film and Photo League Filmography... Jump Cut: A Review of Contemporary Media 17:23-25. Consultado abril 20, 2016, http://www.ejumpcut. org/archive/onlinessays/JC14folder/FPhotoFilogy.html

Böger, Astrid. 2001. People's Lifes, Public Images: The New Deal Documentary Aesthetic. Tubinga: Gunter Narr Verlag.

Bourdieu, Pierre. 1995. Las reglas del arte: génesis y estructura del campo literario. Barcelona: Anagrama.

Campbell, Russell. 1978. "Potamkin's Film Criticism". Jump Cut: A Review of Contemporary Media n. ${ }^{\circ}$ 18: 23-24. Consultado abril 20, 2016. http://www.ejumpcut.org/archive/onlinessays/ JC18folder/HarryAlanPotamkin.html

- 1982. Cinema Strikes Back: Radical Filmmaking in the United States 1930-1942. Ann Arbor: University of Michigan Press.

Carpiolo, Ettore. 1968. El Group Theatre de Nueva York: 1931-1941. Buenos Aires: Editorial Universitaria de Buenos Aires.

Clarck, Katerina. 2000. The Soviet Novel: History as Ritual. Bloomington: Indiana University Press.

Corbus Bezner, Lili. 1999. Photography and Politics in America: From the New Deal into the Cold War. Baltimore: John Hopkins University Press.

Costa, José Manuel. (1999). "Joris Ivens and the Documentary Project". En Joris Ivens and the Documentary Context, editado por Kees Bakker, 16-24. Ámsterdam: Amsterdam University Press.

Denning, Michael. 1996. The Cultural Front: The Labouring of American Culture in the Twentieth Century. Nueva York: Verso.

Ellis, Jack C. y Betsy A. McLane. 2005. A New History Of Documentary Film. Nueva York: Continuum.

García López, Sonia. 2013. Spain is us: la guerra civil española en el cine del Popular Front (19361939). Valencia: Universitat de València.

Hurwitz, Leo. 1934. "The Revolutionary Film, Next Step". New Theatre 3, n. ${ }^{\circ} 6: 14-15$.

— 1975. "One Man's Voyage: Ideas and Filmss in the 1930's." Cinema Journal 1, n. ${ }^{\circ}$ 15:1-15.

Ivens, Joris. 1936. "Notes on Hollywood". New Theatre n. ${ }^{\circ}$ 3:8-10.

Ivens, Joris. 1999. "Notes on the Avantgarde Documentary Film". En Joris Ivens and the Documentary Context, editado por Kees Bakker, 224-226. Ámsterdam: Amsterdam University Press.

James, David E. 2005. The Most Typical Avant-Garde: History and Geography of Minor Cinemas in Los Angeles. Los Ángeles: University of California Press.

Kazan, Elia y Michel Ciment. 1987. Elia Kazan por Elia Kazan. Madrid: Fundamentos.

McCann, Sean. 2000. Gumshoe America: Hard-Boiled Crime Fiction and the Rise and Fall of New Deal Liberalism. Durham: Duke University Press.

Nichols, Bill. 1972. “The American Photo League". Screen 13, n. ${ }^{4}$ 4:108-115. 
Pudovkin, Vsevolod. 1960. Lecciones de cinematografía. Madrid: Rialp.

Rabinowitz, Paula. 1991. Labor \& Desire: Women's Revolutionary Fiction in Depression America. Chapel Hill: University of North Carolina Press Books.

- 1994. They Must Be Represented: The Politics of Documentary. Nueva York: Verso.

Ribalta, Jorge, ed. 2011. El movimiento de la fotografía obrera (1926-1939): ensayos y documentos. Madrid: MNCARS/TF Editores.

Ross, Steven. 1999. "Struggles for the Screen: Workers, Radicals, and the Political Uses of Silent Film". The American Historical Review 96, n. ${ }^{\circ}$ 2:333-367.

Schoots, Hans. 2000. Living Dangerously: A Biography of Joris Ivens. Ámsterdam: Amsterdam University Press.

Schwartz, Nancy Lynn. 1982. The Hollywood Writers' Wars. Nueva York: Knopf.

Sexton, Jamie. 2008. Alternative Film Culture in Inter-War Britain. University of Exeter Press.

Sproule, Michael J. 1997. Propaganda and Democracy: The American Experience of Media and Mass Persuasio. Cambridge: Cambridge University Press.

Staiger, Janet. 1979. “Dividing Labor for Production Control: Thomas Ince and the Rise of the Studio Sistem". Cinema Journal 18, n. ${ }^{\circ}$ 2:16-25.

Steiner, Ralfph y Leo Hurwitz. 1935. "A New Approach to Film Making". New Theatre: 22-23.

Stott, William. 1986. Documentary Expressión and Thirties America. Chicago: University of Chicago Press.

Sweet, Fred, Eugene Rosow, Allan Francovich y Tom Brandon. 1973. "Pioneers: An Interview with Tom Brandon." Film Quarterly 1, n. ${ }^{\circ}$ 27:12-24.

Szalay, Michael. 2000. New Deal Modernism: American Literature and the Invention of the Welfare State. Durham: Duke University Press.

\section{Cómo citar este artículo:}

Granell, Ana Rodríguez. 2017. "No art without propaganda: la constitución de NYKino contra la institución documental" Cuadernos de Música, Artes Visuales y Artes Escénicas 12 (1): 47-61. doi:10.11144/javeriana.mavae12-1.nawp. 